05

\title{
Прочность пластин монокристаллического кремния для солнечных элементов
}

\author{
() В.В. Шпейзман, В.И. Николаев, А.О. Поздняков, А.В. Бобыль, Р.Б. Тимашов, А.И. Аверкин \\ Физико-технический институт им. А.Ф. Иофрфе РАН, \\ 194021 Санкт-Петербург, Россия \\ e-mail: shpeizm.v@mail.ioffe.ru; nkvlad@inbox.ru
}

Поступило в Редакцию 3 апреля 2019 г.

В окончательной редакции 9 апреля 2019 г.

Принято к публикации 13 мая 2019 г.

Рассмотрены способы измерения прочностных характеристик хрупких материалов при осесимметричном изгибе на примере монокристаллического кремния, полученного кристаллизацией из расплава по методу Чохральского, который в виде тонких $(80-200 \mu \mathrm{m})$ пластин используется в большинстве высокоэффективных солнечных элементов с КПД более $20 \%$. Дан анализ экспериментальных и расчетных методов определения напряжений. Сравнены данные численного расчета напряжений с экспериментальными, полученными рентгеновским методом по измерению межплоскостного расстояния в кристаллической решетке кремния под нагрузкой. Показано, что известные формулы теории упругости для расчета напряжений и прогиба справедливы только при нагрузках, много меньших разрушающей нагрузки. Получены зависимости нагрузка-прогиб под нагружающим кольцом при осесимметричном изгибе тонких кремниевых пластин разного размера при различной геометрии испытаний и определена их прочность в зависимости от вида финишной обработки поверхности, которая существенно меняет прочность.

Ключевые слова: монокристаллы кремния, осесимметричный изгиб, расчет напряжений, прочность.

DOI: 10.21883/JTF.2020.01.48665.148-19

\section{Введение}

Интерес к фотоэлектрическим преобразователям энергии, основным материалом для которых является кремний, не ослабевает уже несколько десятилетий. В последние годы пристальное внимание уделяется монокристаллическому кремнию, полученному кристаллизацией из расплава по методу Чохральского, так как такие высококачественные кристаллы обеспечивают высокий КПД солнечных ячеек (solar cell) [1-4]. Одной из важнейших проблем при использовании кремния в преобразователях энергии и других приборах электроники является получение достоверных сведений о его прочности. Поскольку здесь кремний обычно находит применение в виде тонких пластин, то наиболее удобным способом определения его прочности является изгиб, одноосный, когда образцами служат полоски, или двуосный для круглых или квадратных пластин. С другой стороны, прочность хрупких материалов, к которым можно отнести кремний при испытаниях до $900-1000^{\circ} \mathrm{C}$, сильно зависит от состояния поверхности. Так, прочность кремния после химической полировки пластин в смеси азотной и плавиковой кислот может возрасти в десятки и даже сотни раз по сравнению с прочностью пластин после резки или механической полировки [5]. Связано это с тем, что химическая полировка сглаживает поверхностный рельеф в отличие от механической, которая выравнивает поверхность, но практически не изменяет остроту мелких выступов на ней и оставляет высокой величину концентраторов на- пряжений при нагружении. Края пластины или полоски даже после химической полировки содержат более опасные дефекты, чем их плоская поверхность. Поэтому при трех- или четырехточечном изгибе полосок, несмотря на привлекательность анализа линейного напряженного состояния по сравнению с двуосным, не удается достичь столь высоких значений прочности, которые получаются при осесимметричном изгибе круглых пластин. Последний метод иначе называют „кольцо в кольцо“ (ring-on-ring test), поскольку опора и нагружающий пуансон имеют контакт с образцом по окружности. Сказанное выше делает осесимметричный изгиб наиболее достоверным способом определения истинной прочности кремниевых пластин. Однако при его использовании для тонких пластин возникают трудности при анализе напряженного состояния и расчете величины прочности.

В рамках классической теории упругости задачи осесимметричного изгиба круглых пластин с различными условиями на опоре решены давно, и их результаты можно найти, например в [6]. Диаметр пластины в этих решениях совпадал с диаметром опорного кольца. В реальном случае диаметр пластины всегда больше диаметра опоры, при этом тангенциальные напряжения на ее контуре малы (радиальные напряжения на краю пластины равны нулю). В [7] было показано, что вынесенный за опору край пластины не сильно сказывается на величине напряжений, и формула для радиальных $\sigma_{r}$ и равных им тангенциальных $\sigma_{\theta}$ напряжений в области внутри малого круга, где они являются максимальными, 
выглядит следующим образом:

$$
\sigma_{r}=\sigma_{\theta}=\frac{3(1+v)}{2 \pi h^{2}}\left(\ln \frac{b}{a}+\frac{1-v}{1+v} \frac{b^{2}-a^{2}}{2 c^{2}}\right) F,
$$

где $v-$ коэффициент Пуассона, $2 b$ и $2 a-$ соответственно диаметры опоры и нагружающего пуансона, $c$ - радиус и $h-$ толщина пластины, $F-$ величина нагрузки. Отличие формулы (1) от аналогичной для стандартного решения, в котором диаметр пластины равен диаметру опоры $(c=b)$, при небольшом выступающем за опору крае, не приводит к значительному изменению расчетных напряжений.

Рассматривая прогиб пластины, обычно вычисляют его максимальную величину, т.е. в центре пластины. Однако для сравнения с экспериментом удобней пользоваться прогибом в точках окружности с радиусом $r=a$, так как именно этот прогиб $w(a)$ равен перемещению подвижного штока испытательной машины при нагружении. Нетрудно вычислить его, пользуясь моделью, изложенной в [7]:

$$
w(a)=\frac{F}{8 \pi D}\left(\left(b^{2}-\alpha^{2}\right)\left(1+\frac{1-v}{1+v} \frac{b^{2}}{2 c^{2}}\right)+2 a^{2} \ln \frac{a}{b}\right),
$$

где $D=E h^{3} / 12\left(1-v^{2}\right), E-$ модуль упругости. Следует заметить, что формулы (1) и (2) справедливы для изотропного материала, каким можно считать поликристаллический кремний и монокристаллический с ориентацией [111], поскольку в плоскостях $\{111\}$ модуль упругости и коэффициент Пуассона не зависят от направления [8]. Для других ориентаций в [5] было предложено использовать средние интегральные значения $\bar{E}=1 / 2 \pi \int_{0}^{2 \pi} E(\varphi) d \varphi$ и $\bar{v}=1 / 2 \pi \int_{0}^{2 \pi} v(\varphi) d \varphi$. Еще одно допущение было принято в [7] для квадратных пластин. Предлагалось считать величину с равной радиусу равновеликого круга, т.е. равного по площади испытываемой квадратной пластине. По данным других источников использовались следующие соотношения: $c=l(1+\sqrt{2}) / 4$, где $l$ - длина стороны квадрата [9] или $2 c=0.54\left(l_{1}+l_{2}\right)$, где $l_{1}$ и $l_{2}$ - длины сторон для прямоугольной пластины [10]. Разница в формулах для определения $c$ представляется несущественной, особенно если учесть, что, как правило, член в формулах (1) и (2), который содержит $c$, мал по сравнению с другими слагаемыми.

Формула (2) позволяет провести экспериментальную проверку описанного выше расчета прогиба и тем самым установить границу применимости расчетной модели и формулы (1). Целью настоящей работы было получение зависимостей нагрузка-прогиб при осесимметричном изгибе тонких кремниевых пластин разного размера при различной геометрии испытаний и проведение анализа возможности определения достоверных значений прочности известными численными и экспериментальными методами.

\section{1. Методика эксперимента, образцы}

Для исследования были выбраны пластины кремния, используемые в производстве в качестве подложек для серийных солнечных элементов. Подложки кремния толщиной $195 \mu \mathrm{m}$ были нарезаны алмазной проволокой из монокристаллического слитка p-типа диаметром $200 \mathrm{~mm}$, выращенного методом Чохральского в направлении [100]. Слитки подвергались квадратированию, для чего у них были отрезаны 4 сегмента на концах взаимно перпендикулярных диаметров с ориентацией [100], так что в сечении получался квадрат со скругленными вершинами и стороной $156 \mathrm{~mm}$. Пластины такой конфигурации используется в большинстве солнечных панелей. Все пластины испытывались в двух состояниях: исходном и после стравливания нарушенного слоя с последующей пассивацией (толщина последних составляла $175 \mu \mathrm{m})$.

Прочностные испытания пластин проводились на разработанных оригинальных автоматизированных установках осесимметричного изгиба. Пластины помещались на подставку в виде кольца диаметром $60 \mathrm{~mm}$ и нагружались со скоростью $0.2 \mathrm{~mm} / \mathrm{min}$. Оконечная часть штока испытательной машины также представляла кольцо диаметром $20 \mathrm{~mm}$, соосное с первым кольцом и образцом. В ходе эксперимента фиксировалась сила $F$ как функция перемещения штока испытательной машины $\Delta l=w(a)$, т.е. прогиба пластины под нагружающим кольцом.

Чтобы уменьшить величину прогиба при испытаниях изгибом тонких пластин, обычно переходят к малым размерам самой пластины и опорного, и нагружающего колец. Так, в [11] диаметры колец были 4.5 и $9 \mathrm{~mm}$, a пластины кремния $10 \times 10 \mathrm{~mm}$ имели толщину от 300 до $1000 \mu \mathrm{m}$. Нами также были проведены испытания подложек монокристаллического кремния с ориентацией [100] малого размера толщиной $0.1 \mathrm{~mm}$ при следующей геометрии изгиба: $2 a=4.4,2 b=8.4$, $2 c=11.8 \mathrm{~mm}$. Эти пластины были двух типов, отличавшихся видом финишной обработки поверхности: а) химическим травлением и б) механической полировкой.

\section{2. Результаты экспериментов и их обсуждение}

На рис. 1 приведены примеры зависимостей $F(\Delta l)$ травленых (рис. $1, a$, кривая 1 ) и нетравленых (рис. $2, b$, кривая 1) образцов большого диаметра. Там же показаны прямые, вычисленные по формуле (2). В результате было обнаружено, что примерно до прогиба, равного $50 \mu \mathrm{m}$, функция $F(\Delta l)$ близка к точному решению осесимметричной задачи теории упругости с усредненными по различным направлениям в (100) модулем упругости и коэффициентом Пуассона, которая в нашем случае предсказывает зависимость $F=3.9 \Delta l$ для нетравленых и $F=2.8 \Delta l$ для травленых пластин 



Рис. 1. Соотношение между нагрузкой $(F)$ и прогибом (перемещением нагружающего кольца $\Delta l$ ) при осесимметричном изгибе $(a)$ химически травленых пластин кремния большого размера и исходных после резки (нетравленых) $(b): 1-$ эксперимент, 2 - расчет по формуле (2). На врезке показан начальный участок зависимости $F(\Delta l)$.

( $F$ в $\mathrm{N}$ и $\Delta l$ в $\mathrm{mm})$. При $\Delta l>50 \mu \mathrm{m}$, что соответствует $F \approx 0.25 \mathrm{~N}$, сила начинает резко возрастать, т.е. формула (2) показывает бо́льшие значения прогиба, чем реальные Это означает, что расчет напряжений по формуле (1) дает при указанной геометрии испытаний завышенные значения напряжений, начиная уже с рассчитанных по (1) напряжений $\sigma \approx 5 \mathrm{MPa}$. Причиной этого могут служить различные факторы: мембранный эффект при изгибе [7], большой угол поворота пластины на опоре, а также неупругие эффекты, связанные с зарождением дислокаций в приповерхностном слое, которое наблюдалось в [12] и приводило к разрушению при циклическом нагружении изгибом даже при комнатной температуре.

Результаты испытаний пластин малого диаметра показаны на рис. 2. Из рисунка следует, что отклоне- ния от (2) начинаются при $\Delta l>250 \mu \mathrm{m}$ и $F \approx 7 \mathrm{~N}$. Эти значения выше, чем в предыдущем случае, но все равно далеки от величин нагрузки и прогиба в момент разрушения. Из сказанного следует, что при расчете прочности тонких пластин методом „кольцо в кольцо“ формулу (1) можно использовать только в области малых напряжений. К такому выводу пришли авторы работы [5] при сравнении вычислений напряжений по (1) с непосредственными измерениями упругих деформаций рентгеновским методом с последующим расчетом компонент тензора напряжений [5]. Для этого в нижней опоре под образцом было сделано отверстие, через которое под заданным углом направлялся рентгеновский луч. Основы метода изложены в [13]. Рентгеновский метод дает возможность дистанционно, без нанесения каких-либо датчиков или реперов исследовать распределение деформации на поверхности монокристаллов путем измерения углов дифракции рентгеновского пучка, направленного в различные точки поверхности. Интенсивные максимумы с хорошо разрешенным $K_{\alpha}$-дублетом позволяют при измерениях на монокристаллах использовать тонкий рентгеновский луч и локализовать область измерения в пределах $0.2-0.3 \mathrm{~mm}$. Применение дифракции рентгеновских лучей для определения напряжений основано на том, что вследствие упругой деформации кристалла изменяется межплоскостное расстояние и, следовательно, угол дифракции. Определяя изменение угла дифракции при разных нагрузках и для плоскостей с различной ориентацией, можно восстановить полный тензор деформаций и затем рассчитать компоненты тензора напряжений.

В [5] приведены примеры полученных зависимостей максимальных напряжений от нагрузки при различной геометрии испытаний, а также распределение радиальных и тангенциальных напряжений по радиусу образца. Для образца кремния ориентации [111] после

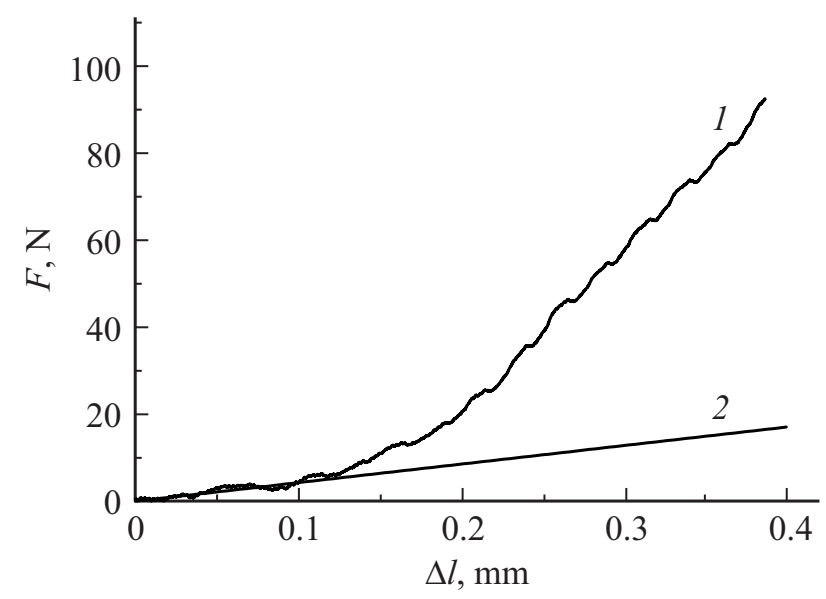

Рис. 2. Соотношение между нагрузкой $(F)$ и прогибом $(\Delta l)$ при осесимметричном изгибе пластин кремния малого диаметpa: 1 - эксперимент, 2 - расчет по формуле (2). 


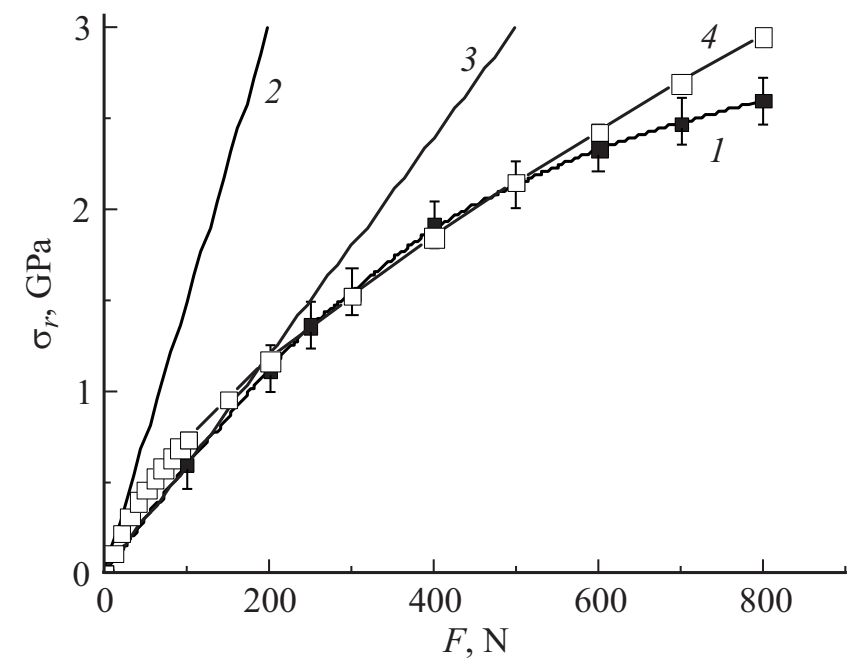

Рис. 3. Радиальные напряжения в пластине кремния. 1 экспериментальная зависимость максимальных радиальных напряжений $\sigma_{r}$ в пластине кремния от усилия при нагружении „кольцо в кольцо“, полученная рентгеновским методом [5,13], 2 - расчетная прямая по формуле (1) для $h=0.22,2 a=6$, $2 b=18,2 c=30 \mathrm{~mm}, 3-$ линейная зависимость для оценки напряжений при использованных в настоящей работе условий опыта, 4 - расчет напряжений по методу конечных элементов (FEM).

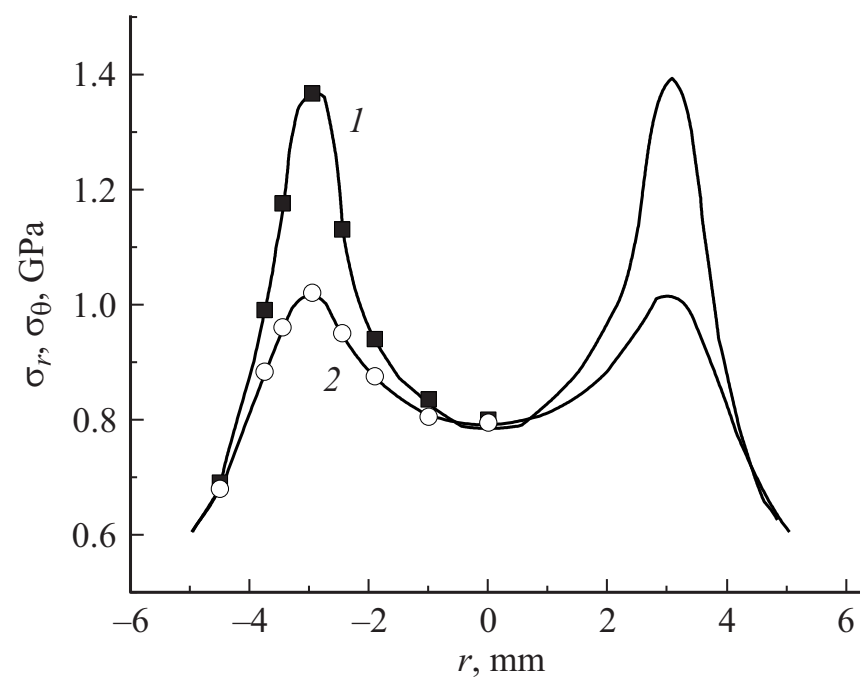

Рис. 4. Распределения радиальных $\sigma_{r}$ и тангенциальных $\sigma_{\theta}$ напряжений по радиусу в пластине кремния при нагружении „кольцо в кольцо“, полученные рентгеновским методом $[5,13]$, $h=0.22,2 a=6,2 b=18,2 c=30 \mathrm{~mm}, F=250 \mathrm{~N}$.

химической полировки и геометрии испытаний: $2 a=6$, $2 b=18,2 c=30$ и $h=0.22 \mathrm{~mm}$, зависимость $\sigma_{r}(F)$ показана на рис. 3. Она расположена значительно ниже прямой, рассчитанной по формуле (1), дающей в нашем случае совершенно не реальные значения прочности, которая, как видно из рис. 3, для лучших образцов достигала $2.7 \mathrm{GPa}$. Распределение радиальных и танген-
Сила в момент разрушения пластин кремния с $(a)$ химической и $(b)$ механической финишной обработкой поверхности $F_{\mathrm{fr}}$ и определенные по ней максимальные радиальные напряжения в момент разрушения с использованием формулы (1) $\sigma_{\mathrm{cal}}$, приближенной оценки по рентгеновским измерениям $\sigma_{\text {est }}$, а также по данным расчетов методами FDM $\sigma_{\mathrm{FDM}}[14]$ и FEM $\sigma_{\mathrm{FEM}}$

\begin{tabular}{c|c|c|c|c|c}
\hline Серия & $F_{\text {fr }}, \mathrm{N}$ & $\begin{array}{c}\sigma_{\text {cal }}, \\
\mathrm{GPa}\end{array}$ & $\begin{array}{c}\sigma_{\text {est }}, \\
\mathrm{GPa}\end{array}$ & $\begin{array}{c}\sigma_{\mathrm{FEM}}, \\
\mathrm{GPa}\end{array}$ & $\begin{array}{c}\sigma_{\mathrm{FDM}}, \\
\mathrm{GPa}\end{array}$ \\
\hline$a$ & $71.5 \pm 36.1$ & $3.18 \pm 1.60$ & $1.12 \pm 0.45$ & 1.17 & 0.96 \\
$b$ & $28.9 \pm 11.5$ & $1.29 \pm 0.51$ & $0.51 \pm 0.20$ & 0.63 & 0.50
\end{tabular}

циальных напряжений по радиусу (рис. 4) для точки c $F=250 \mathrm{~N}$ на кривой $\sigma_{r}(F)$ (рис. 3) также не соответствует формуле (1). Радиальные напряжения больше тангенциальных и непостоянны внутри малого кольца. Радиальные и тангенциальным напряжения равны только в центре пластины, где они равны по определению. Максимальными оказываются напряжения под малым кольцом. В масштабе осей на рис. 3 не видно области, которая адекватно описывается формулой (1), уже первая экспериментальная точка находится далеко за ее пределами. Рентгеновский метод измерения напряжений можно использовать не только для нагружения „кольцо в кольцо“, но и для случая „шарик в кольцо“, для которого нельзя получить формулы, подобные формулам (1) и (2), поскольку при сделанных при их выводе предположениях напряжения в центре пластины обращаются в бесконечность. Примеры тарировочных кривых для такого способа нагружения приведены в [5].

Недостатком рентгеновского метода определения напряжений является то, что для каждой геометрии испытаний и размеров образца нужно получать свою тарировочную кривую. Однако для ориентировочных оценок и близких условий испытания можно использовать корреляционные соотношения. Так, например, на рис. 3 проведена прямая 2, которая близка к реальной кривой до $F \sim 200 \mathrm{~N}$, а наклон ее в 2.5 раза меньше рассчитанной по формуле (1). Предполагая, что коэффициент 2.5 сохранится для условий испытания малых образцов с размерами и условиями испытания, описанными выше, можно оценить их прочность $\sigma_{\text {est. }}$. В таблице представлены результаты такой оценки прочности кремниевых пластин с различной финишной обработкой поверхности ( $a$ и $b)$. Всего было испытано 50 образцов. Несмотря на большой разброс значений прочности отчетливо видно преимущество химического травления поверхности пластин над ее механической обработкой в плане увеличения прочности. Можно отметить, что даже при малых напряжениях формула (1) не находит экспериментального подтверждения для гибких пластин. Очевидно, ее можно использовать только для достаточно толстых образцов, напряженное состояние 
которых можно описать изгибом с нейтральной осью в середине сечения.

Расчеты различных случаев осесимметричного изгиба тонких пластин численными методами в рамках нелинейной теории упругости получили распространение вскоре после появления первых ЭВМ. В [14] приведены результаты вычислений напряжений и прогиба в пластинах различной формы и с разными условиями нагружения методом конечных разностей (finite difference method (FDM)). Они показывают, что формулами (1) и (2) можно пользоваться только в области очень малых напряжений и прогибов. Из таблиц и рисунков, приведенных в [14], следует также, что при достаточно больших нагрузках радиальные и тангенциальные напряжения при $r<a$ различны по величине и зависят от расстояние от центра. Нами было проведено сравнение численных расчетов прочности с экспериментальными для двух случаев геометрии нагружения, описанных выше. Первый - сравнение с условиями рентгеновских измерений, второй - с оценкой напряжений разрушения для пластин кремния малого размера. Кроме анализа данных [14], в настоящей работе было проведено конечно-элементное (finite element method (FEM)) моделирование системы [14] в пакете Comsol Multiphysics. Рассматривалась осесимметричная модель пластины с применением прямоугольных конечных элементов и характерным размером ячейки примерно в 4 раза меньшей толщины пластины. В качестве граничных условий задавались отсутствие перемещений по периметру соприкосновения опорного кольца и пластины и равномерное распределение нагрузки по периметру соприкосновения нагружающего кольца и пластины. Использованная модель обеспечивала хорошую сходимость решения. Как следует из рис. 3, расчет напряжений для показанной на нем геометрии испытаний кремниевых пластин хорошо согласуются с данными рентгеновских измерений. Сравнение приближенной оценки прочности $\sigma_{\text {est }} \mathrm{c}$ рассчитанной по данным [14] и FEM для подобных условий испытания, показывает, что $\sigma_{\text {est }}$ близка к результатам расчета по обоим методам (см. таблицу).

\section{Заключение}

Важность получения достоверных значений прочности тонких пластин кремния, как и путей ее увеличения, не вызывает сомнений. Ясно также, что осесимметричный изгиб является наилучшим из существующих способом испытания тонких пластин. Прямое измерение упругих напряжений или деформаций, например рентгеновским методом, сопряжено с большими трудностями из-за сложности эксперимента и неудобством при исследовании пластин малого размера. Контактные методы определения напряжений создают дефекты на поверхности и могут вызвать более раннее разрушение. Надежность расчетных методов, особенно для прочных пластин с большим прогибом, должна быть подтверждена прямыми или косвенными экспериментами. Одним из вопросов в расчетах является, насколько предполагаемые при расчетах условия на опоре соответствуют реальным. В качестве одного из способов проверки расчета напряжений может быть сравнение расчета и эксперимента для прогиба пластины. Это не требует ни дополнительных устройств, ни специальных экспериментов, достаточно только при измерения прочности записывать величину силы и перемещения. Совпадение расчета прогиба с экспериментальным его значением может служить основанием для того, чтобы доверять аналогичному расчету для напряжений.

\section{Благодарности}

Выражаем благодарность сотрудникам Solar Silicon Technology (г. Подольск) Е.В. Смирновой и С.Т. Даветадзе за предоставленные образцы.

\section{Конфликт интересов}

Авторы заявляют, что у них нет конфликта интересов.

\section{Список литературы}

[1] Fertig F., Krauß K., Rein S. // Energ. Proced. 2017. Vol. 124. P. 338.

[2] Danilewsky A., Wittge J., Kiefl K., Allen D., McNally P., Garagorri J., Elizalde M.R., Baumbach T., Tanner B.K. // J. Appl. Cryst. 2013. Vol. 46. P. 849.

[3] Gabor A.M., Janoch R., Anselmo A., Lincoln J.L., Seigneur H., Honeker Ch. // IEEE J. Photovoltaics. 2016. Vol. 6. N 1. P. 3575 .

[4] Rozgonyi G., Youssef K., Kulshreshtha P., Shi M., Good E. // Solid State Phenomena. 2011. Vol. 178-179. P. 79. doi: 10.4028/www.scientific.net/SSP.178-179.79

[5] Степанов В.А., Песчанская Н.Н., Шиейзман В.В. Прочность и релаксационные явления в твердых телах. Л.: Наука, 1984. 245 с.

[6] Тимошенко С.П., Войновский-Кригер С. Пластины и оболочки. М.: Наука, 1966. $635 \mathrm{c}$.

[7] Витман Ф.Ф., Уфлянд Я.С., Иоффе Б.С. // Прикладная механика. 1970. Т. 6. № 5. С. 122.

[8] Най Д. Физические свойства кристаллов. М.: ИЛ, 1960. $385 \mathrm{c}$.

[9] Coletti G., van der Borg N.J.C.M., De Iuliis S., Tool C.J.J., Geerligs L.J. rx06032. 21st European Photovoltaic Solar Energy Conference and Exhibition, 4-8 September 2006, Dresden, Germany.

[10] ASTM C1499-15. Standard Test Method for Monotonic Equibiaxial Flexural Strength of Advanced Ceramics at Ambient Temperature. 
[11] Gouttebroze S., Lange H.I., Ma X., Glockner R., Emamifard B., Syvertsen M., Vardavoulias M., Ulyashin A. // Phys. Stat. Solid. 2013. A Vol. 210. N 4. P. 777-784.

[12] Жога Л.В., Степанов В.А., Шпейзман В.В. // ФТТ. 1977. Т. 19. Вып. 8. С. 1521

[13] Васильев Д.М., Титовеи Ю.Ф. // Заводская лаборатория. 1977. Т. 43. № 10. С. 1235.

[14] Корнишин М.С., Исанбаева Ф.С. Гибкие пластины и панели. М.: Наука, 1968. 258 с. 